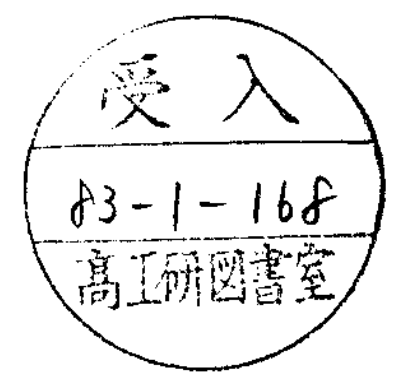

Ref.TH. 3473-CERN

THE COSMOLOGICAL CONSTANT IN SPONTANEOUSLY COMPACTIFIED

\title{
$D=11$ SUPERGRAVITY
}

\author{
M.J. Duff *) \\ CERN - Geneva \\ and \\ C.A. Orzalesi \\ Istituto di Fisica dell'Università, Parma \\ and INFN, Sezione di Milano
}

\section{A B S T R A C T}

\begin{abstract}
Spin-torsion mechanisms, which produce spontaneous compactification with vanishing cosmological constant in four dimensions, are discussed within $D=11$ supergravity.
\end{abstract} *) On leave from The Blackett Laboratory, Imperial
College, London.

Ref.TH.3473-CERN

25 November 1982 


\section{.}


The field equations of $N=1$ supergravity in $D=11$ dimensions 1 ) admit solutions in which seven of the dimensions are spontaneousiy compactified ${ }^{2)}$. A particular solution describes the product of four dimensional anti-deSitter (AdS) space-time with the seven-sphere $S^{7}$ and yields an effective four-dimensional theory with $\mathrm{N}=8$ supersymmetry, explicit so(8) gauge invariance and other hidden symmetries ${ }^{3)}$. The massless sector may probably be identified with the local SO(8) $\times S U(8)$ theory of Ref. 4 . Other solutions with the same $S^{7}$ topology but different geometry have also been found 5),6). These new solutions reinforce the hopes of finding a realistic superunified field theory, since they provide a Kaluza-Klein origin for the Higgs and super-Higgs effect in four dimensions 6).

It is remarkable that such a dynamical compactification to four dimensions, and the corresponding symmetry breaking, should be automatically built into the $D=11$ theory, and this is to be contrasted with the trivial dimensional reduction schemes, where one imposes from the outside in a somewhat ad hoc manner a particular dependence of the fields on the extra co-ordinates F see, for example, Ref. 7)].

However, there is a serious objection to spontaneously compactified supergravity, and that is the appearance of an enormous cosmological constant. In non-supersymmetric theories admitting spontaneous compactification, one could add a cosmological term to the $D>4$ Lagrangian, so as to arrange for a cancellation of the one arising in $D=4$, but no such modification of $D=11$ supergravity is allowed. (Even in the non-supersymmetric case, this is a somewhat dubious procedure and would not be stable against renormalization effects.)

In this paper we discuss a possible cure for this problem, based on the observation already made in generalized Kaluza-Klein theories ${ }^{8)}$ that fermionic bilinears can provide a non-vanishing torsion tensor, which can be used to flatten the connection in the extra compact dimensions provided the "internal" space is parallelizable. The curvature of the compact internal space now vanishes for the full connection, even though the Riemann curvature is non-trivial, and this leads ${ }^{8)}$ to a vanishing cosmological constant for $D=4$ space-time : $\Lambda=0$.

The motivation for this paper began with the following three observations :

(i) the crucial ingredient for $\Lambda=0$ is parallelizability ;

(ii) the beauty of the $S^{7}$ spontaneous compactification of $D=11$ supergravity derived largely from the fact that $S^{7}$ admits a Cartan-Shouten parallelism 9) (i.e., it is a non-product manifold which not only is parallelizable 
but it has flat connections with totally antisymmetric torsion and, indeed, it is the only non-group compact simply connected manifold with this property ;

(iii) the torsion tensor of $D=11$ supergravity ${ }^{1)}$ is described by two terms $(M, N, A=I, \ldots, I I)$

$$
C_{M N A}=\frac{i}{2} \bar{\Psi}_{B} \Gamma_{M N A}{ }^{B D} \Psi_{D}-i \bar{\Psi}_{M} \Gamma_{A} \Psi_{N},
$$

where the first term is totally antisymmetric while the second term is antisymmetric only in $M$ and $N$; consequently, one might conjecture that the first term should play the dominant rôle in parallelizing $\mathrm{S}^{7}$; this conjecture seems to be supported also by the fact that a CartanShouten parallelizing torsion can indeed be expressed in the form of the first term in Eq. (1), provided one uses for $\psi_{D}$ suitably chosen zeromodes of the $D=11$ supergravity on $S^{7}$.

However, our results turn out to be just opposite to such expectations: the first term in (1) actually cancels against other terms in the $D=11$ Lagrangian, and never makes its appearance in the field equations (this fact might even be guessed on grounds of supercovariance). Since the first term in (1) is immaterial for a spin-torsion compactification with $\Lambda=0$, we are led to consider the possible effects of the second term in (1) and, consequently, we are led to broaden our scope and to include manifolds which, though still parallelizable, are made curvature-flat by a non-totally antisymmetric torsion. One possibility is the solution of the $D=11$ field equations considered in Ref. 3), namely $S^{5} \times S^{2}$ It is interesting to note that this manifold fulfils the witten ${ }^{10}$ ) criterion : it leads to a four-dimensional theory with gauge group $\mathrm{SU}(4) \times \mathrm{SU}(2)$, containing $\mathrm{SU}(3) \times \mathrm{SU}(2) \times U(1)$. It is also interesting that the choice $S^{5} \times S^{2}$ would be uniquely selected if one were to require that (a) it is a solution, made out of (products of) spheres, of the classical $D=11$ field equations, and (b) it does not admit a parallelizing torsion which is totally antisymmetric in any group of three indices: indeed, (a) restricts the choice to Einstein internal spaces, and hence excludes $S^{3}$ factors, and (b) excludes $S^{4} \times S^{3}$ and $S^{7}$.

Now let us discuss the cosmological constant problem. The field equations of $D=11$ supergravity are 11 )

$$
\Gamma^{R S T} \hat{D}_{S}(\hat{\omega}) \Psi_{T}=0,
$$


$-3-$

$$
\begin{aligned}
& D_{T}(\hat{\omega}) \hat{F}^{T U R S} \\
& \quad=\frac{1}{(24)^{2}} \varepsilon^{M N P Q V W X X U S R} \hat{F}_{M N P Q} \hat{F}_{U W X Y}, \\
& R_{T S}(\hat{\omega})-\frac{1}{2} g_{T S} R(\hat{\omega}) \\
& \quad=\frac{1}{24}\left[g_{T S} \hat{F}_{M N P Q} \hat{F}^{M N P Q}-8 \hat{F}_{M N P T} \hat{F}^{M N P}\right],
\end{aligned}
$$

where

$$
\begin{aligned}
& \hat{D}_{S}(\hat{\omega}) \Psi_{T}=D_{S}(\hat{\omega}) \Psi_{T}+T_{S}^{M N P Q} \hat{F}_{M N P Q} \Psi_{T}, \\
& \hat{\omega}_{M R S}=\omega_{M R S}(e)+\frac{i}{2}\left(2 \bar{\Psi}_{M} \Gamma_{[S} \Psi_{R]}+\bar{\Psi}_{S} \Gamma_{M} \Psi_{R}\right), \\
& \hat{F}_{M N P Q}=F_{M N P Q}-3 \bar{\Psi}_{[M} \Gamma_{N P} \Psi_{Q]}, \\
& F_{M N P Q}=4 \partial_{[M} A_{N P Q],} \\
& T^{S M N P Q}=\frac{1}{(12)^{2}}\left(\Gamma^{S M N P Q}-8 \Gamma^{[M N P} \eta^{Q] S}\right) .
\end{aligned}
$$

[ Our conventions are those of Refs. 1) and 11).]

The conventional ansatz for the ground state solution, consistent with $D=4$. Lorentz invariance, would be to set the fermion fields to zero and

$$
\begin{aligned}
& \left\langle g_{\mu \nu}\right\rangle=g_{\mu \nu}(x),\left\langle g_{m n}\right\rangle=g_{m n}(y),\left\langle g_{\mu m}\right\rangle=0, \\
& \left\langle F_{\mu \nu \rho \sigma}\right\rangle=f_{\mu \nu \rho \sigma}(x)=\frac{c}{\left.\left.\right|^{4} g\right|^{1 / 2}} \varepsilon_{\mu \nu \rho \sigma}, \\
& \left\langle F_{m n p q}\right\rangle=f_{m n p q}(y),
\end{aligned}
$$




$$
\left\langle F_{\mu \nu \rho p}\right\rangle=\left\langle F_{\mu \nu p q}\right\rangle=\left\langle F_{\mu n p q}\right\rangle=0,
$$

where Greek indices run over space-time with co-ordinates $x^{\mu}$ and Latin indices over the extra dimensions with co-ordinates $\mathrm{y}^{\mathrm{m}}$, and where $c$ is a constant and $f_{\mu \nu \rho \sigma}$ and $f_{m n p q}$ must satisfy the classical field equation obtained from (3) by setting the fermion bilinears equal to zero and using $\left\langle\mathcal{E}_{\mathrm{MN}}\right\rangle$ in place of $\mathrm{E}_{\mathrm{MN}}$. bilinears, yields 6)

Substituting (10) into the field equation (4), with vanishing fermion

$$
\begin{aligned}
& R_{\mu}^{\mu}(e)=-\frac{1}{36}\left[8 F_{4}^{2}-4 F_{7}^{2}\right] \geqq 0, \\
& R_{m}^{m}(e)=-\frac{1}{36}\left[-7 F_{4}^{2}+5 F_{7}^{2}\right] \leqq 0,
\end{aligned}
$$

where

$$
\begin{aligned}
& F_{4}^{2} \equiv f_{\mu \nu \rho \sigma} f^{\mu \nu \rho \sigma} \leqq 0, \\
& F_{7}^{2} \equiv f_{m n p q} f^{m n p q} \geqq 0 .
\end{aligned}
$$

It then follows that no choice of $g_{\mu \nu}(x), g_{m n}(y)$ or $f_{m n p q}(y)$ will lead to $\mathrm{R}_{\mu}^{\mu}(e)=0$, as required for vanishing $\Lambda$, except the trivial case $(c=0$, $f_{\text {mnpq }}=0$ ). Of course, this case is realized in the Cremmer-Julia theory of Ref. 1I), which corresponds to a trivial dimensional reduction on a seven-torus. However, it is also clear that, with Eqs. (10), a non-trivial spontaneous compactification with $\Lambda=0$ cannot occur unless we admit the possibility of nonvanishing torsion in the ground state, i.e., non-vanishing expectation values for fermion bilinears. [Note that, as we anticipated, the totally antisymmetric term in Eq. (1) has disappeared from the field equations.] Four-dimensional Lorentz invariance permits

$$
S_{m s x}(y) \equiv \frac{i}{2}\left\langle\bar{\Psi}_{m} \Gamma_{s} \Psi_{x}\right\rangle \neq 0
$$


but the other components of $\left\langle\Psi_{M} \Gamma_{S} \Psi_{R}\right\rangle$ must vanish. While allowing for $S_{m n r}(y) \neq 0$, we shall not commit ourselves to any specific mechanism by which fermion bilinears might acquire non-zero expectation values consistent with four-dimensional Lorentz invariance : we have in mind a quantum condensate phenomenon ${ }^{8}$ ), but they might also arise from classical fermion field configurations. In fact, in this respect, our results will be model-independent.

Notice that, in particular,

$$
\left\langle\Psi_{\mu} \Gamma_{\nu} \Psi_{\rho}\right\rangle=0 \text {, }
$$

hence the connection $\hat{\omega}_{\mu}^{\alpha \beta}(x)$ of space-time will be torsion-free : $\hat{\omega}_{\mu}^{\alpha \beta}(x)=$ $=\omega_{\mu}^{\alpha \beta}(e)$. The requirement that space-time be Minkowski space $(\Lambda=0)$ then leads immediately to the vanishing of the $\mathrm{d}=4$ energy-momentum tensor $\left\langle\mathrm{T}_{\mu \nu}\right\rangle$.

Consequently,

$$
\left\langle\hat{F}_{M N P Q}\right\rangle=0
$$

and $\left\langle\mathrm{T}_{\mathrm{MN}}\right\rangle=0$. Note from $\mathrm{Eq}$. (7) that this does not necessarily imply vanishing $\left\langle\mathrm{F}_{M N P Q}\right\rangle$, because Lorentz invariance in four dimensions allows for non-vanishing values for some of the bilinears in (7); we can allow for

$$
\begin{aligned}
& \left\langle\bar{\Psi}_{[\mu} \Gamma_{\nu_{\rho}} \Psi_{\sigma]}\right\rangle=\frac{c}{\left.\left.3\right|^{4} g\right|^{1 / 2}} \varepsilon_{\mu \nu \rho \sigma}, \\
& \left\langle\bar{\Psi}_{[m} \Gamma_{n p} \Psi_{q]}\right\rangle=\frac{1}{3} f_{m n p q}(y),
\end{aligned}
$$

while the $\mu n p q, \mu v p q, \mu v \rho q$ components of $\left\langle\Psi\left[\mathrm{M}_{\mathrm{NP}} \Psi_{Q}\right]^{\rangle}\right.$must vanish.

We also note that (15) is a supercovariant statement and that it is consistent with Eq. (3) as well as with the other field equations.

We are now left with

$$
R_{\mu \nu}(e)=0
$$




$$
R_{m n}(\hat{\omega})=0
$$

where now

$$
\hat{\omega}_{m r s}=\omega_{m r s}(e)+S_{m r s}-S_{m r s}+S_{s m r} .
$$

But (19) can be solved by choosing $S_{\text {mrs }}$ to be a parallelizing torsion on the compact internal manifold $\left.{ }^{*}\right)$, in which case

$$
R_{m n p q}(\hat{\omega})=0
$$

Thus we have arrived at a four-dimensional theory with zero cosmological constant whenever the seven-dimensional internal compact space can be parallelized by the torsion $S_{\text {msr }}$. A question which we have not yet answered is what happens to supersymmetry in this approach. If in the gravitino field transformation law,

$$
\delta \Psi_{M}=D_{M}(\hat{\omega}) \epsilon+T_{M}^{P Q R S} \hat{F}_{P Q R S} \epsilon,
$$

we set $\hat{\mathrm{F}}_{\mathrm{PQRS}}=0$, then we obtain

$$
\left[D_{M}(\hat{\omega}), D_{N}(\hat{\omega})\right] \epsilon=\frac{1}{4} R_{M N A B}(\hat{\omega}) \Gamma^{A B} \epsilon=0
$$

as a consequence of Eq. (2I). One might expect that Eq. (22) implies the existence of eight covariantly constant spinors, and hence $N=8$ unbroken supersymmetry. Indeed, one would mimic what happens in Riemann-flat manifolds : one would pick a parallelization, i.e., a moving frame $e_{A}{ }^{M} \partial_{M}$ such that $\hat{\omega}_{A B C}$ vanishes in this frame, so as to rewrite $\delta \Psi_{M}=0$ as $e_{A}{ }_{M} \partial_{M} \varepsilon=0$, with eight solutions given by spinors which are $\mathrm{y}$ independent in the given moving frame. This argument suggests that supersymmetry might be unbroken but it is not clear that the

*)

An alternative way to obtain $\Lambda=0$ would be to allow for an internal space which is Ricci-flat $\left[R_{m n}(e)=0\right]$ but not Riemann-flat $\left[R_{m n r s}(e) \neq 0\right]:$ in such cases, a vanishing ground-state torsion is allowed. We discard this posquently, lead for additional details. 
background parallelization remains invariant under supersymmetry transformation. A more reasonable expectation is that the maximum number of unbroken supersymmetries should be determined by the maximum invariance group allowed for the parallelizing torsion $\underset{\sim}{\hat{\omega}}$. This, in turn, might depend on the details of the geometry of internal space. In particular, it would be interesting to know how many (if any) supersymmetries can remain unbroken when $S^{7}$ is parallelized by a non-totally antisymmetric torsion.

We also note that the fact that $\langle\hat{F}\rangle$ can be vanishing with non-vanishing $\langle\underset{\sim}{F}$, see Eqs. (10) and (16)-(17), is perhaps suggestive of a phase in which $\langle\underset{\sim}{\mathrm{F}}\rangle \neq 0$ triggers the compactification with $\Lambda \neq 0$, as in Refs. 2) and 3), and a transition to a spin-condensate phase, in which a parallelizing torsion is generated $(\Lambda=0)$, together with the cancellation of $\langle\underset{\sim}{F}\rangle$ through (16) and (17). The corresponding final situation, where $\Lambda=0$, might be stable against renormalization effects (contrary to what happens ${ }^{12}$ ) in ordinary gravity) because there is an underlying (spontaneously broken) local supersymmetry.

A final remark is that non-vanishing torsion in the vacuum affects 8) the tree-approximation fermion mass spectrum. This might be relevant, in an approach of the kind advocated in Ref. 10), in connection with the chirality problem for "realistic" spinor zero-modes, as well as in other approaches, of the kind discussed in Ref. 13), where one hopes for a realistic spectrum for composite particles. In particular, torsion effects might be relevant also in the $\mathrm{D}=4$, $S O(8)$ theory of Ref. 4), and should be taken into consideration.

\section{ACKNOWLEDGEMENTS}

We thank S. Ferrara and L. Girardello for discussions. 


\section{REFERENCES}

1) E. Cremmer, B. Julia and J. Scherk - Phys.Letters $76 \mathrm{~B}$ (1978) 409 ;

E. Cremmer and B. Julia - Nuclear Phys. B159 (1979) 141.

2) P.G.O. Freund and M.A. Rubin - Phys.Letters 94B (1980) 233.

3) M.J. Duff and C.N. Pope - to appear. Reported by M.J. Duff in "Supergravity 81", Eds. S. Ferrara and J.G. Taylor, Cambridge University Press (1982) and by M.J. Duff and D.J. Toms in "Unification of the Fundamental Interactions II", Eds. S. Ferrara and J. Ellis, Plenum Press (1982), CERN Preprint TH. 3259 (1982).

4) B. de Wit and H. Nicolai - Phys.Letters 108B (1982) 285 and CERN Preprint TH. 3291 (1982).

5) F. Englert - CERN Preprint TH. 3394 (1982).

6). M.J. Duff - CERN Preprint TH. 3451 (1982)

7) E. Cremmer - in "Supergravity 81", Eds. S. Ferrara and J.G. Taylor, Cambridge University Press (1982).

8) C.A. Orzalesi and M. Pauri - Phys.Letters 107 B (1981) 186 ; C. Destri, C.A. Orzalesi and P. Rossi - New York University Preprint TR8/82
(1982).

9) E. Cartan and J.A. Schouten - Proc.K.Akad.Wet.Amsterdam 29 (I926) 933.

10) E. Witten - Nuclear Phys. B186 (1981) 412.

11) E. Cremmer and S. Ferrara - Phys.Letters 91B (1980) 61.

12) S.M. Christensen and M.J. Duff - Nuclear Phys. B170 (1980) 480.

13) J. Ellis, M.K. Gaillard, L. Maiani and B. Zumino - in "Unification of the Fundamental Interactions", Eds. S. Ferrara, J. Ellis and

P. van Nieuwenhuizen, Plenum Press (1980). 